\title{
Option einer ambulanten Zwangsbehandlung - Kontra
}

\author{
egislation for Compulsory Outpatient Psychiatric Treatment - Contra
}

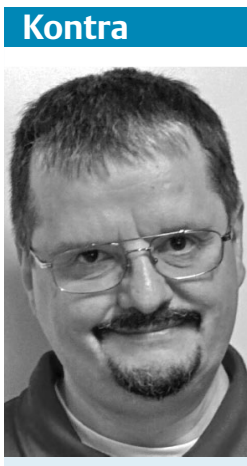

Rainer Höflacher
Immer wieder wird in Fachkreisen die Möglichkeit der ambulanten Zwangsbehandlung diskutiert. Im Jahr 2003 wurde in Deutschland erwogen, diese im Betreuungsrecht gesetzlich zu legitimieren. Es entstand aber eine politische Mehrheit, die dazu führte, dass ein gefährdeter Patient weiterhin nur stationär und bei fehlender Einwilligungsfähigkeit gegen seinen Willen behandelt werden darf. Trotzdem lebt der diesbezügliche Diskurs immer wieder auf. In England gibt es die Möglichkeit, Patienten ambulant zur Behandlung zu zwingen. Auflagen können sein, in die Sprechstunden zu kommen, Urinuntersuchungen auf Drogenmissbrauch zuzulassen, Hausbesuche zu erlauben und Psychopharmaka einzunehmen. Dieses Vorgehen kann auf Jahre angeordnet werden. Obwohl es wissenschaftlich umstritten ist, dass ambulante Zwangsbehandlung Vorteile bringt und obwohl es fraglich ist, ob sie den Ansprüchen der UN-Menschenrechtskonvention entspricht, wird in England häufig davon Gebrauch gemacht. Die Befürworter der ambulanten Zwangsbehandlung halten diese für hilfreich und legitim, da ein kleiner Kreis von psychisch erkrankten Menschen immer wieder in eine psychische Krise gerät, wenn die Medikamente abgesetzt werden. Bei diesen Patienten entstehen dann regelmäßig Situationen der Fremd- oder Eigengefährdung, mit denen dann auch Straftaten einhergehen können. Es komme häufig vor, dass das Gefährdungspotenzial der Betroffenen rapide ansteige, wenn keine Psychopharmaka mehr eingenommen werden, so wird häufig argumentiert. Es sei nun für diese wenigen Patienten das weniger schädliche Vorgehen, wenn sie per Auflage dazu gezwungen werden am- bulant Medikamente einzunehmen, um so einer Zwangseinweisung mit eventueller stationärer Zwangsmedikation zu entgehen. Die Einschränkungen, die der Patient dabei in Kauf nehmen müsse, seien deutlich geringer als bei einer zwangsweisen stationären Unterbringung, die Wochen oder vielleicht sogar Monate dauern könne. Das sei ein humanes Handeln, da ein stationärer Aufenthalt vermieden werden könne und dem Patienten grundsätzlich Schlimmeres erspart bliebe. Zudem käme so die Psychiatrie ihrem Ordnungsauftrag angemessen nach.

Tatsächlich zeigt sich hier aber der Widerspruch zwischen gut gemeinter ärztlicher Fürsorge und der Verletzung wesentlicher Freiheitsrechte. Es ist zu bedenken, dass die ambulante Zwangsbehandlung an Menschen ausgeführt werden soll, die momentan einwilligungsfähig und nicht fremd- oder eigengefährdet sind. Diese haben genauso wie der Rest der Bevölkerung das Recht selbst darüber zu entscheiden, ob sie Nachteile haben werden oder nicht. Das Menschenrecht auf Freiheit und Selbstbestimmung darf in diesem Fall nicht eingeschränkt werden. In den letzten Jahren hat sich die Rechtsprechung vermehrt dahingehend entwickelt, dass Patienten selbst entscheiden dürfen, ob sie gesundheitlich Schaden nehmen oder nicht. Auch das neue Patientenverfügungsgesetz, das die Wirksamkeit einer Patientenverfügung garantiert, ist ein Schritt in diese Richtung. Diese „Freiheit zur Krankheit“" muss auch für psychisch erkrankte Menschen gelten, die einwilligungsfähig sind und keine Gefahr für sich oder andere darstellen, unabhängig davon, ob mit hoher Wahrscheinlichkeit eine Gefährdung oder Verschlechterung des Gesundheitszustands zu erwarten ist. Im ersten Moment scheint der Grad Einflussnahme auf den Patienten hinnehmbar. Denn es scheint sich ja „nur“ um einen indirekten Zwang zu handeln. Aber eine Auflage, bei deren Verletzung keine Konsequenzen zu erwarten sind, hat keine Überzeugungskraft. Sollte der Patient der Vorschrift durch den Arzt nicht nachkommen, kann dies zu körperlicher Gewalt führen, wenn es folgerichtig zur Zwangseinweisung kommt. Denn wie anders kann der Psychiater gerichtlichen Zwangsauflagen Gewicht verleihen, als bei Nichtbefolgung entsprechenden Zwang eben gerichtlich oder letztlich polizeilich einzufordern?

Die Gefahr ist sehr groß, dass die Möglichkeit, Menschen zur Medikamenteneinnahme zu zwingen, missbraucht wird. Zum Beispiel können Hilflosigkeits- und Ohnmachtsgefühle der Ärzte schnell dazu führen, dass das Instrument der ambulanten Zwangsbehandlung viel zu schnell eingesetzt wird, obwohl es noch andere Möglichkeiten gäbe, zu einer Lösung zu kommen. Es sollte ein ärztliches Grundanliegen sein, den gesundheitlichen Zustand eines psychisch erkrankten Menschen zu bessern. Es hat sich gezeigt, dass eine vertrauensvolle Arzt-Patient-Beziehung hierfür eine große Wirksamkeit hat. Die Anwendung von Zwang ist kurzfristig oft eine einfache Lösung, aber langfristig behindert sie den Prozess der Genesung und vermindert die Einflussmöglichkeiten der Behandler. Was vom Arzt zwar als Hilfe gemeint sein mag, faktisch vom Betroffenen aber als Drohung und Erpressung wahrgenommen wird, bringt den Arzt dauerhaft von der Rolle des Helfers in die einer Kontrollinstanz. Wie soll auf diesem Boden eine vertrauensvolle und offene, nicht von untergründigem Misstrauen geprägte Beziehung noch möglich sein?

In der Bevölkerung herrschen immer noch Bedenken und Ängste gegenüber psychiatrischer Behandlung, die ja aufgrund der deutschen Geschichte nicht unberechtigt sind. Seit vielen Jahren wird versucht, den Bürgern das humanitäre Anliegen der Psychiatrie-Fachpersonen zu vermitteln, um diesen so wirksamer helfen zu können. Eine Psychiatrie, die nicht mit Druck und Zwang arbeitet, kann zu einem Hilfesystem werden, das auf Vertrauen und Wohlwollen gegründet 
ist. Die Möglichkeit, Patienten gesetzlich ambulant zur Medikamenteneinnahme zu zwingen, wie dies z.B. in den Niederlanden geplant ist, würde uns zurückwerfen und es bestünde die Gefahr, dass Zwang nicht nur bei den Betroffenen, sondern auch in der öffentlichen Wahrnehmung wieder $\mathrm{zu}$ einem bestimmenden Merkmal psychiatrischer Behandlung würde. Dies wäre ein sehr hoher Preis für die Möglichkeit, bei einigen wenigen Personen in eine möglicherweise schädliche Entwicklung eingreifen zu können. Ich glaube kaum, dass die psychiatrischen Professionellen dies wollen können. Die Betroffenen jedenfalls wünschen sich eine solche Psychiatrie nicht.

Fazit: Im Grundsatz müssen zwei widersprüchliche Sichtweisen gegenübergestellt werden. Einerseits die Möglichkeit Schaden abzuwenden, indem die Betrof- fenen zur Medikamenteneinnahme ambulant gezwungen werden, und so Gefahr und Leiden eventuell vermieden werden können. Andererseits stehen dem die Selbstbestimmungs- und Freiheitsrechte der Betroffenen gegenüber. Je nachdem, aus welcher Interessenslage man an diesen Widerspruch herangeht, kann die Lösung anders aussehen. Die Gefahr besteht darin, dass bei der Legitimierung der ambulanten Zwangsbehandlung das Zwangsprinzip unkontrolliert in die Psychiatrie wieder Eingang findet. Dies wäre ein Rückschritt. So ist es sinnvoll, dass weiterhin bei einer psychischen Erkrankung nur Fremd- oder Eigengefährdung zu einer Unterbringung führen können. Inwieweit dann eine eventuell sich anschließende stationäre Zwangsbehandlung letztendlich dem Patienten dienlich ist, bleibt ebenso umstritten.
Sie haben eine eigene Meinung zu diesem

Thema? Dann schreiben Sie uns an:

psychiat-praxis@thieme.de!

Korrespondenzadresse

\section{Rainer Höflacher}

Geschäftsführer des Landesverbandes

Psychiatrie-Erfahrener Baden-Württemberg e. V. Schlosserstraße 28a

70180 Stuttgart

hoeflacher@lvpebw.de

Bibliografie

Dol http://dx.doi.org/

10.1055/s-0034-1370003

Psychiat Prax 2014; 41: 240-241

(c) Georg Thieme Verlag KG

Stuttgart · New York

ISSN 0303-4259 